\title{
A Multiple Constrained QoS Routing Algorithm for the wireless sensor networks
}

\author{
Zhou Zeshun , Xu Yi , Yan Junjie , Nie Zhongwei , Li Layuan \\ School of Computer Science and Technology, Wuhan University of Technology \\ Wuhan,China 430063
}

\begin{abstract}
Routing problem is one of the most important issues to a wireless sensor network (WSN). It is the key problem to find an efficient energy strategy for prolonging network's lifetime because power supply might be impossible. This paper discusses the multicast routing problem of WSN with multiple QoS constraints, which may deal with the delay, bandwidth, hop count and packet reception rat and surplus energy metrics, and finds a minimum resource consumption path while satisfying multiple constraints optimization conditions, and describes a network model for researching the multicast routing problem. It presents a dynamic multicast routing algorithm with multiple QoS constraints (MCQoSRA). The MCQoSRA successfully solves the QoS routing problems when multicast nodes change dynamically in the networks. The MCQoSRA only requires the local state information of the link (or node), but does not require any global network sate to be maintained. In MCQoSRA, a multicast group member can join or leave the multicast session dynamically. The MCQoSRA can effectively decrease the overhead for constructing a multicast tree and the delay of the nodes, and improve the success ratio of seeking links. Simulation results show that the MCQoSRA provides an available means to implement multicast routing, and adapt to all kinds of the topology networks, and have better expansibility.
\end{abstract}

\section{Keywords- WSN; QoS; multicast routing; energy;}

\section{INTRODUCTION}

The wireless sensor networks will enable the reliable monitoring of a variety of environments such as battlefields and disaster fields. Traditional routing protocols do not take into account that a node contains only a limited energy supply. Optimal routing tries to maximize the duration over which the sensing task can be performed. Sensors are typically disposable and expected to last until their energy drains due to their battery constrained power. The energy constrained and limited computing resources of the sensor nodes present major challenges in gathering data.

In the wireless sensor network, the node excess energy, the wireless band width, the hops of the nodes, the time delay and the time delay vibration are the main parameter of reflecting wireless sensor network essence characteristic. And the node excess energy displays especially essential.

Therefore, how to make highly and effectively use of the energy to maximization network life cycle is the most important challenge which the wireless sensor network faces. Along with the application of the wireless sensor network is widespread, demands of transmitting the multimedia service in the wireless sensor are promoted unceasingly, and the correspondence quality requests are also higher and higher. QoS multicasts technology becomes a key technology of transmitting information in the network.

The goal of QoS multicast routing technology is to find an algorithm or Strategy of seeking a link mode, in a given network and multicast demand situation, the cyber source can be effectively.

A dynamic QoS multicast routing protocol (DQMRP) was presented in [1], but it did not take into consideration the energy problem. SEQMRA-W ${ }^{[2]}$ based on maximal nodes' surplus energy is maximum residual energy QoS multicast routing algorithm, and takes maximization network lifetime as a goal, embarks from the WSN node residual energy and the available bandwidth. QPSO $^{[3]}$ (quantum particle swarm optimization algorithm) solved the QoS multicast routing by reducing energy consumption and ensure QoS in data transmission of the WSN.

In this paper, we abbreviate a Dynamic Multicast Routing Algorithm with Multiple QoS Constrained as MCQoSRA. MCQoSRA can successfully find the route that can satisfy required QoS constraints, if such a path exists. At the same time, it could effectively distribute the traffic throughout the network, while adding a new node to the existing multicast tree. At the same time, we study the delay, the hops, and packet reception-constrained low energy consumption QoS multicast routing problem which is known to be NP-complete, a multicast group member can join or leave a multicast session dynamically. It can significantly reduce the overhead of constructing a multicast tree. The join of new member can have minimum overhead to other on-tree nodes or non-tree nodes, and only requires minimum adjustment to original tree and minimum energy reservation process. It can also minimized the overall cost of the tree, and satisfy multiple QoS constraints and least energy's (or lower energy) requirements.

\section{NETWORK MODEL}

A wireless sensor network can be modeled as a weighted digraph $G=(V, U)$, where $V$ is the set of nodes, representing routers or switches, $U$ denotes the set of communication links connecting the nodes. Let $s \in V$ be source node of a multicast tree, and $M \subseteq\{V-\{s\}\}$ be a set of end nodes of the multicast tree. Let $R$ be the positive weight and $R^{+}$be no negative weight. For any Link $e \in U$, we can define the some QoS metrics: delay function 
delay $(e), U \rightarrow R$; energy consumption energy $(e), U \rightarrow R^{+}$; bandwidth function bandwidth $(e), U \rightarrow R^{+}$, and hop counts function hop $(e), U \rightarrow R^{+}$; and inherent total energy function allenergy $(e), V \rightarrow R^{+}$, packet reception rate function reception $(e), V \rightarrow R^{+}$and inherent total energy function allenergy $(e), V \rightarrow R^{+}$, we also use $T(s, M)$ to denote a multicast tree ${ }^{[4]}$. Therefore they are defined as follows:

$$
\begin{aligned}
& \operatorname{delay}\left(\mathrm{P}_{\mathrm{T}}(\mathrm{s}, \mathrm{k})\right)=\sum_{e \in P(s, k)} \operatorname{delay}(e) \\
& \operatorname{bandwidth}\left(\mathrm{P}_{\mathrm{T}}(\mathrm{s}, \mathrm{k})\right)=\min \{\operatorname{bandwidth}(\mathrm{e})\} \\
& \operatorname{hop}\left(\mathrm{P}_{\mathrm{T}}(\mathrm{s}, \mathrm{k})=\sum_{e \in P(s, k)} \operatorname{hop}(e)\right. \\
& \operatorname{hop}(e)=1, e \in P ; \text { hop }(e)=0, e \notin P \\
& \operatorname{reception}\left(\mathrm{P}_{\mathrm{T}}(\mathrm{s}, \mathrm{k})\right)=\prod_{n \in P(s, k)} \text { reception }(e) \\
& \text { allenergy }(\mathrm{P})=\frac{\operatorname{delay}(\mathrm{P}) \times \operatorname{hop}(\mathrm{P})}{\operatorname{bandwidth}(\mathrm{P}) \times \operatorname{reception}(P)}
\end{aligned}
$$

$P(s, k)$ denotes the path from source $s$ to end node $k$ of $T(s, M)$.

Definition1: MCQoSRA is intended for multicast applications. Where $P(s, k)$ denotes the path from source $\mathrm{s}$ to end node $\mathrm{k}$ of $T(s, M)$. QoS-based multicast routing problem deals mainly with some elements: Network $G=(V, U)$, multicast source $s \in V$, the set of end nodes $M \subseteq\{V$ - $\{s\}\}$, delay $(\cdot) R$, energy $(\cdot) R^{+}$, bandwidth $(\cdot) R^{+}$, and hop $(\cdot) R^{+}$. This routing problem is to find the $T(s, M)$ which satisfies some QoS constraints:

$$
\begin{aligned}
& \operatorname{delay}\left(\mathrm{P}_{\mathrm{T}}(\mathrm{s}, \mathrm{k})\right) \leq \mathrm{D}_{\text {max }} \\
& \operatorname{bandwidth}\left(\mathrm{P}_{\mathrm{T}}(\mathrm{s}, \mathrm{k})\right) \geq \mathrm{B}_{\text {min }} \\
& \operatorname{reception}\left(\mathrm{P}_{\mathrm{T}}(\mathrm{s}, \mathrm{k})\right) \geq \mathrm{R}_{\text {min }}
\end{aligned}
$$

The optimization objective is allenergy $(P)$. If the above conditions are satisfied, allenergy $(P)$ is minimum energy consumption. Bmin is the bandwidth restrain, Dmax is the delay restrain and Rmin is the packet reception restrain.

$$
\text { Definition2:If } \quad P(s, t) \quad \text { satisfied }
$$
$\left(\mathrm{d}\left(\mathrm{s},{ }^{*}\right)+\mathrm{d}(\mathrm{k}, \mathrm{t}) \leq \mathrm{D}_{\max }\right) \Lambda\left(\mathrm{b}(\mathrm{i}, \mathrm{j}) \geq \mathrm{B}_{\min }\right) \Lambda \quad(R(P(s, t)))=\min \left[r_{1}, \ldots, r_{f}\right]$, then should meet the minimum path for energy consumption. The minimum energy path multicast tree of the meeting the bandwidth and delay constraints is a NP-complete problem, and it can adopt some heuristic algorithm to solve.

\section{MCQoSRA}

\section{A. The strategy of the nodes' selection}

Multicast node join operation involves adding point, generally it has three selection strategies of random dots, the delay and minimum energy consumption. The random dots strategy from the multicast tree is either a routing node as a multicast destination entry point, the minimum delay strategy is $\mathrm{s}$ to $\mathrm{d}$ minimum delay path routing and multicast sub-trees intersection as multicast destination $d$ join points, the minimum energy strategy is the selection of from $\mathrm{s}$ to $\mathrm{d}$ minimum energy consumption routing path and multicast sub-trees intersection as multicast destination $d$ adding point ${ }^{[5]}$. The simulation results show that the minimum energy consumption, strategy cost the best precision, so MCQoSRA selects the minimum energy strategies.

\section{B. MCQoSRA description}

According to the characteristics of the wireless sensor network, the goal of the QoS routing optimization is to select the following conditions and to satisfy the Minimum path of the energy consumption.

Meanwhile, the energy ( $T(s, M)$ should be minimum. Where $D_{\max }$ is delay constraint, $B_{\min }$ is bandwidth constraint.

Let $G=(V, E)$ be a network, where $V=\left\{v_{1}, \ldots, v_{\mathrm{n}}\right\}$ is the set of nodes in $G$ and $E=\left\{e_{1}, \ldots, e_{\mathrm{m}}\right\}$ is the set of links in $G$. $v_{\mathrm{i}}=$ (node_delay, node reception), node delay is the delay of the node and node_ reception is the packet reception rate of the node. $e_{\mathrm{j}}=$ (link_energy, link bandwidth, link delay), link energy,is the link energy consumption, link_bandwidth is the link bandwidth, link delay is the link delay. Consider a QoS request $R=(S$, $\left.D, \bar{B}, R, R_{\min }\right)$, where $S$ and $D$ are the source node and the destination node, respectively, $B$ is the bandwidth requirement, $D$ is the delay requirement, $C$ the minimum energy requirement. The goal is for each QoS request $R$, it constructs a routing path satisfying the request when such a routing path exists, or report that no such a path exists. The algorithm is presented as follows.

To any $G=(V, E), v_{\mathrm{i}}=$ (node delay, node reception), $e_{\mathrm{j}}=$ (link_energy, link_bandwidth, link_delay), $R=(S, D, B$, $R, C)$, compute the link path $P$ from the source node to the destination node and satisfy the following condition:

$$
\begin{aligned}
& \text { Link_bandwidth }(j) \geq B, j \in P \\
& \sum_{i \in P} \text { node_delay(i) } \sum_{i \in P} \text { node_delay }(j) \leq D_{\max } \\
& \prod_{i \in P} \text { node_reception }(i) \geq R_{\min } \\
& \sum_{j \in P} \text { link_energy }(j) \leq R_{\min }
\end{aligned}
$$

\section{MCQoSRA implementation}

The basic idea of MCQoSRA is to use available link bandwidth and delay constraints to construct multicast routing tree for satisfying the minimum energy consumption. This come from $\mathrm{BIP}^{[6]}$ minimum spanning tree Prim algorithm the basic idea to form. The source node is the root node, first find the node of a minimum energy consumption is added to the tree, and then BIP uses the minimum energy increment principle every time to add a node in the tree, finally all nodes added to the tree, the tree is a minimal energy multicast tree.MCQoSRA first selects the multicast source to consist of initialization of the multicast tree, then requests or exits according to the multicast member connection, in accordance with the operating rules of the entry and exit, dynamically creates or disconnects. Multicast tree formation is a process of dynamic multicast members joining and exiting. In MCQoSRA, router or node must be maintained for some specific information in the routing table. In order to describe conveniently, the main news of the MCQoSRA 
routing and node can be defined as: Request indicates the message of the joining request, the node send from t to multicast source $\mathrm{s}$, and requests to join the multicast tree; Accept indicates the confirmation joined message, the source node sends the request authorization from $\mathrm{s}$ to $\mathrm{t}$, and confirms the joining request; Delete indicates the message of the link deletion, the node reverses transmission along the multicast tree from $t$ to the parent node $\mathrm{w}$, and request to remove the $\operatorname{link}(W, t)$; Establish indicates the establishing state, the routing resource in the node has been assigned.

MCQoSRA builds the multicast tree by a distributed way for each node with the same algorithm, four different nodes ( the source node, a node on the tree, the intermediate node and a target node ) exchanges information in different ways, each node is triggered by reaching the control message ( Request, Accept, Delete and Establish ). Bandwidth, delay and energy in link e are $B(e), D(e), R(e)$, and $r$ table for the local routing table, for any node $v, r$ table $[v] . \bar{b}$ expressed in the shortest path on the useful bandwidth, $r$ table[v].d expressed in the shortest path on the useful delay, $r$ table $[v] . R$ expressed in the shortest path on the useful energy. Therefore, MCQoSRA can be described as the main procedure ${ }^{[7]}$ :

Step1. Remove any links with $B<B_{\min }$ gain a new $G_{1}$;

Step2. Multicast source node $s$ radio Request message, began to search links;

Setp3. When a node i receives a Request, if Request has already been received and processed, then transmits Request message to its adjacent node; or if $\operatorname{delay}(p$ $(u, v))>D_{\max }$, then pointer poi[u] should record for $u$ parent node;

Step4. Calculation of the minimum energy path;

If a path satisfies the minimum energy definition 2

then poi $[y]=x$

IF $\mathrm{x}$ is Establish then Calculate $\mathrm{d}(P(s, x))$

IF delay $\left(P(s, x) \leq D_{\max }\right.$ then

While poi $[u] \neq$ NIL, then sends Request message;

Step5. If the node $\mathrm{k}$ receives the transmitted Accept message of the node $\mathrm{j}$, then the link $e(j, k)$ is Establish;

Step6. Check whether all multicast destination nodes have joined the multicast tree $T(s, M)$, if not, then the source node $\mathrm{s}$ continues to broadcast Request packets, to execute Step2, or Step7;

Step7. Implementation of the pruning algorithm, using the traversal algorithm traverses above the generated multicast trees based on the multicast source nodes for the root node, and visits the leaf node. If the leaf node is not a multicast node, then deletes the node, until all leaf nodes of the multicast tree are multicast destination node.

\section{CORRECTNESS AND COMPLEXITY ANALYSIS}

\section{A. The correctness proof}

Theorem1. If a path from a new member to $T(s, M)$ has sufficient resources to satisfy multiple QoS constraints and has minimum energy consumption, it searches only one path.

Proof: The proof is straightforward. Because any links, which do not have enough bandwidth available, will be eliminated before running the algorithm. A route found by MCQoSRA will contain such satisfying links. The paths being searched will be marked by the routing entries at the nodes. In MCQoSRA, any routing entry has a single out interface and one or multiple in interfaces. Hence, the nodes will form a searching tree structure. This tree is just a $T(s, M)^{[8]}$.

Theorem2. An available and feasible path found by MCQoSRA is loop free. If MCQoSRA terminates without an available and feasible path, all nodes out of $T(s, M)$ are ether initial state or in failure state.

Proof. MCQoSRA terminates without success only when the new member's joining is rejected, i.e., it changes into the failure state. Since the new member is leaf node of the searching tree, when it changes into the failure state, all nodes in the searching tree must be in the failure state. The nodes outside the searching tree remain in the initial state.

Theorem3:MCQoSRA constructed multicast tree $T(s, M)$ will be able to meet the bandwidth, delay, minimum energy constraint.

Proof . MCQoSRA has calculated the minimum energy path according to definition1 principle; and in MCQoSRA, if and only if $d\left(P(s, v) \leq D_{\max }\right.$ and $\left.\left.b(p)(s, v)\right) \geq B_{\min }\right)$, it issued a join request message, therefore, for $\forall \mathrm{P}(\mathrm{s}, \mathrm{v}) \subseteq \mathrm{T}(\mathrm{s}, \mathrm{M}), P(\mathrm{~s}, v)$ will be able to meet the time delay, bandwidth and energy constrained. So MCQoSRA constructed multicast tree $T(s, M)$ will be able to meet the bandwidth, delay and energy constraints.

\section{B. Complexity Analysis}

The complexity of MCQoSRA can be analyzed in terms of the computation complexity and the number of messages needed to construct a multicast tree. The former mainly concerns the computation overhead needed to find paths and construct the multicast tree. The later mainly involves the overhead of message exchange needed to build the multicast tree. In MCQoSRA, if QoS metrics are delay and bandwidth, the complexity is $O(M+n \log n)$; if QoS metrics are delay, bandwidth, packet loss and energy consumption, the complexity is $O\left(h^{*}(M+n \log n)\right), n$ is the circulate number. where $|V|$ is the number of nodes and $|E|$ is the number of edges in a network. For most networks, $|E|=O|V|$, hence the complexity is $O\left(|V|^{2}\right)$. For a multicast group with $|M|$ members, the computation overhead is $\mathrm{O}\left(|V|^{2}|M|\right)$. YAM and QoSMIC (centralized or distributed) are K.2|M|, K.2|M|.

\section{EXPERIMENTAL RESULTS}

We use the Network Simulator 2 as the basic simulation platform. DMRM is implemented by using NS2 and it's performance is compared with some existing algorithms. Network topologies used in the simulations are deliberately manipulated to simulate wide area sparse networks ${ }^{[9]}$. A large network is likely to be loosely interconnected .An n-node graph is considered to be sparse when less than $5 \%$ of the possible edges are present in the graph. The network graphs used in the simulations are by 
Waxman's random graph mode.

In the simulations, we compare the quality of routing trees by the energy consumption of SEQMRA-W, QPSO and MCQoSRA. The energy consumption is measured by the mean value of the total number of simulation runs. At each simulation point, the simulation runs 80 times. Each time the nodes in the group $\mathrm{G}$ are randomly picked out from the network graph.

Figure 1 shows a multicast tree with multicast nodes energy consumption increased change curve, the simulation experiment network node number is set to 500 , the delay was 300 , when multicast node number increases, the SEQMRA-W, QPSO and MCQoSRA generated by the energy consumption also increased, but MCQoSRA increasing is smaller, the same multicast group MCQoSRA relatively little energy consumption. Since the control message length increases, the energy consumption increases. Therefore, in the network of smaller scenarios routing control messages to bring extra energy consumption offset control mechanism of saving energy, but with the increase in the number of nodes in the network, MCQoSRA energy consumption will be much lower than that of the SEQMRA-W, QPSO.

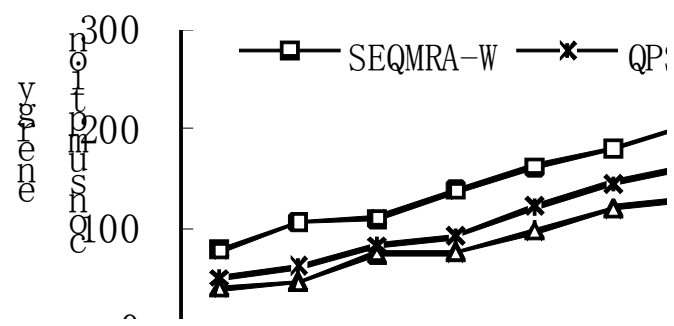

Figure 1: energy consumption

Figure 2 shows the SEQMRA-W, QPSO and MCQoSRA accepting the average success packet rate at any time delay constraint variation curve seen from the graph, MCQoSRA raccepts the average success rate higher than that of the QPSO and MCQoSRA

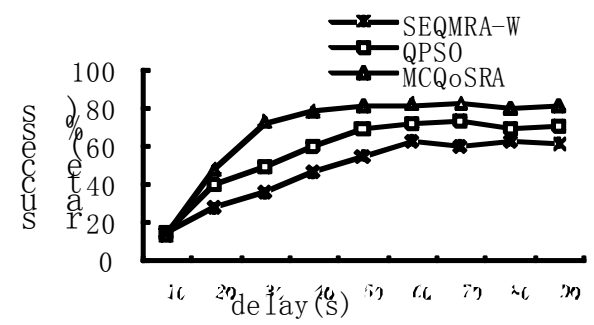

Figure 2: the average success rate

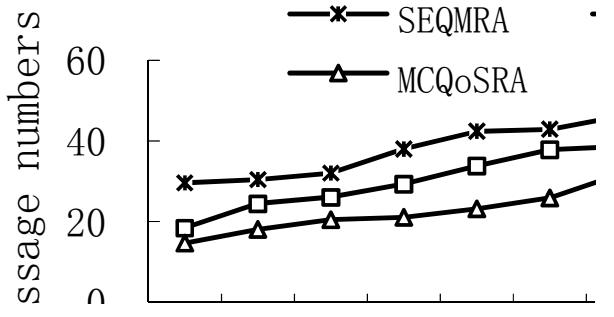

Figure 3 the message number changes

Figure 3 shows the time limit of $100,80,60$, the number of network nodes in [50,500], when MCQoSRA changes, the average number of messages can be seen along with the increase of the number of nodes. The average number of MCQoSRA messages increase very slowly, do not produce news storm, MCQoSRA can be used in large network.

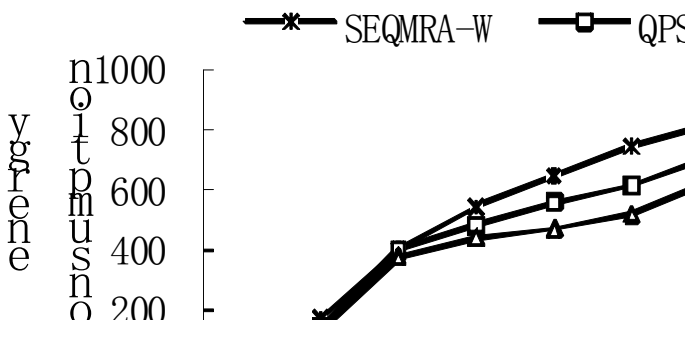

Figure 4 the energy consumption

Figure 4 shows, the energy consumption decreases with the increase of time energy gradually increasing curve, because MCQoSRA the multicast tree nodes dynamically increase or decrease, In the MCQoSRA multicast tree formation process, its contents added value to less.

Based on the SEQMRA-W, QPSO and MCQoSRA, three simulation results compare discovery, MCQoSRA in performance is superior to other two algorithms, which can well satisfy the multiple QoS constraints of wireless sensor network for real-time application.

\section{CONCLUSION}

The paper discusses the multicast routing problem with multiple QoS constraints, which may deal with the delay, bandwidth, packet reception metrics, and energy consumption, and describe a network model for researching the routing problem. We have presented a Multiple QoS constrained Dynamic Multicast Routing Algorithm (MCQoSRA). The MCQoSRA can significantly reduce the overhead of establishing a multicast tree. In MCQoSRA, a multicast group member can join or leave a multicast session dynamically, which should not disrupt the multicast tree. The MCQoSRA also attempts to minimize overall energy consumption of the tree. The join of new member can have minimum overhead to on-tree or non-tree nodes. The correctness 
proof and complexity analysis have been made. Some simulation results are also given. The study shows that MCQoSRA is an available and feasible approach to multicast routing with multiple QoS constraints. Further work will investigate the protocol's suitability for inter-domain multicast and hierarchical network environment.

\section{ACKNOWLEDGMENT}

The work is supported by National Natural Science Foundation of China (61171075) and NSF of Hubei Province(2012FFB05006, 2011CDB297).

\section{REFERENCES}

[1] Li Layuan and Li ChunLin. A Multicast Routing Protocol with Multiple QoS constrains, Proc ofWCC Aug.20021345-1350

[2] YANG Yun,GAO Feng,LIBin,TAO Bi-lei. Based on W SN Network the Largest Remaining Energy QoS Multicast Routing Algorithm. International Forum on Information Technology and Application. 2009,732-736

[3] Huang Yourui, Tian Yiming, Tang Chaoli. Simulation Research of the QoS Multicast Routing in WSN Based on QPSO Algorithm. Third International Symposium on Intelligent Information Technology Application.Third International Symposium on Intelligent Information Technology Application.2009,39-43

[4] Debasish Chakraborty, Goutam Chakraborty,and Norio Shiratori "A Dynamic Multicast Routing Satisfying Multiple QoS Constrains". International Journal of Network Management. Volume13, Issue5 (September 2003).pp.321-335

[5] Huimei Lu, Meilin Shi, Yong Xiang, Min Yang "A New Distributed Scheme for Bandwidth- and Delay-constrained MulticastRouting”.http://cscw.cs.tsinghua.edu.cn/cscwpapers/2002/ICAC T2002-QDMR-tsinghua-finalversion.pdf

[6] Wang Jianxin "QoS Routing Algorithms with Inaccurate Network State Information".Adissertation Submitted for the Degree of Doctor Of Philosophy. October 2001,pp,58 65

[7] Wang Jianxin,Wang Weiping, Chen Jianer,Chen Songqiao. "A Randomized QoS Routing Algorithm On Networks with Inaccurate Link-State Information". Journal of Computer Science and Technology. Volume 17, Issue 1 (January 2002).pp.38-46

[8] Mohamed F. Mokbel Wafaa A. El-Haweet M. Nazih El-Derini "AN EFFICIENT ALGORITHM FOR SHORTEST PATH MULTICAST ROUTING UNDER DELAY AND DELAY VARIATION CONSTRAINTS". www.cs.purdue.edu/ homes/mokbel/ SPECTS2000.pdf

[9] Turgay Korkmaz and Marwan Krunz, "A Dynamic Algorithm for Finding a Path Subject to Multiple QoS Constrains" http://citeseer.nj. nec.com/ 